PHYSICAL REVIEW D 79, 115016 (2009)

\title{
Asymmetric dark matter
}

\author{
David E. Kaplan \\ Physics Department, Johns Hopkins University, Baltimore, Maryland 21218, USA \\ Markus A. Luty \\ Physics Department, University of California, Davis, California 95616, USA
}

Kathryn M. Zurek

Center for Particle Astrophysics, Fermi National Accelerator Laboratory, Batavia, Illinois 60510, USA, and Physics Department, University of Wisconsin, Madison, Wisconsin 53706, USA

(Received 3 March 2009; published 23 June 2009)

\begin{abstract}
We consider a simple class of models in which the relic density of dark matter is determined by the baryon asymmetry of the Universe. In these models a $B-L$ asymmetry generated at high temperatures is transferred to the dark matter, which is charged under $B-L$. The interactions that transfer the asymmetry decouple at temperatures above the dark matter mass, freezing in a dark matter asymmetry of order the baryon asymmetry. This explains the observed relation between the baryon and dark matter densities for the dark matter mass in the range 5-15 GeV. The symmetric component of the dark matter can annihilate efficiently to light pseudoscalar Higgs particles $a$ or via $t$-channel exchange of new scalar doublets. The first possibility allows for $h^{0} \rightarrow a a$ decays, while the second predicts a light charged Higgs-like scalar decaying to $\tau \nu$. Direct detection can arise from Higgs exchange in the first model or a nonzero magnetic moment in the second. In supersymmetric models, the would-be lightest supersymmetric partner can decay into pairs of dark matter particles plus standard model particles, possibly with displaced vertices.
\end{abstract}

DOI: 10.1103/PhysRevD.79.115016

PACS numbers: $95.35 .+\mathrm{d}$

\section{INTRODUCTION}

There is compelling evidence from astrophysical and cosmological data that the dominant contribution of the matter in the Universe is in the form of "dark matter" that interacts very weakly with ordinary matter [1]. One of the striking features of this picture is that the dark matter density today is rather close to the baryon density: $\rho_{\mathrm{DM}} \simeq$ $4.5 \rho_{\text {baryon }}$ in the standard cosmological model [2], suggesting that these relic densities have a common origin. However, in the standard paradigm for dark matter, the dark matter and baryon relic densities arise by completely different mechanisms, and the fact that they have the same order of magnitude is a "cosmic coincidence."

In the standard cosmology the baryon relic density arises from a tiny baryon-antibaryon asymmetry of order $10^{-10}$ at temperatures above $10 \mathrm{MeV}$. This paradigm is strongly supported by the success of big-bang nucleosynthesis. The baryon asymmetry can be generated starting from an initially symmetric universe ("baryogenesis") if the baryon number and $C P$ are violated out of equilibrium in the early Universe [3]. Nonperturbative effects in the standard model efficiently violate baryon and lepton number at temperatures above the electroweak phase transition ( $T \gtrsim 100 \mathrm{GeV})$, so the simplest possibility is that a $B-L$ asymmetry is generated at high scales, e.g. by leptogenesis.

In contrast with the baryon relic density, the origin of the dark matter abundance is not strongly constrained by cosmological data. The most popular model is a weakly interacting massive particle (WIMP) whose relic density is determined by the freeze-out of its annihilations to standard model particles. This naturally explains the observed order of magnitude of the dark matter relic abundance, but not why this is close to the baryon abundance.

In this paper we consider a simple explanation for $\rho_{\mathrm{DM}} \sim \rho_{\text {baryon }}$, namely, that the dark matter density arises from a dark matter particle-antiparticle asymmetry related to the $B-L$ asymmetry. Previous models based on this idea are described in [4-12]. In our models, the $B-L$ and dark matter asymmetries can be related by interactions in equilibrium that transfer the $B-L$ asymmetry (assumed to arise from a standard baryogenesis mechanism) to the dark matter. Any interaction that forces the dark matter to carry a nonzero $B-L$ charge will accomplish this. Unlike most previous models where the $B-L$ number in the dark and standard model sectors were equal and opposite so that the total $B-L$ number of the Universe is zero, in our models there is a net nonzero $B-L$ number that is distributed between sectors. Since the dark matter relic density is set by the baryon asymmetry and not by the properties of thermal freeze-out, we term this class of models asymmetric dark matter (ADM).

This mechanism predicts $n_{\mathrm{DM}} \sim n_{B}$, and therefore $\Omega_{\mathrm{DM}} \sim\left(m_{\mathrm{DM}} / m_{B}\right) \Omega_{B}$. We therefore obtain the observed dark matter abundance for $m_{\mathrm{DM}} \sim 5 \mathrm{GeV}$. The precise dark matter mass is calculable in a given model, and the models we construct give values in the range from 5 to 
$15 \mathrm{GeV}$. These values are close to the electroweak scale, suggesting that the dark matter mass is generated by electroweak symmetry breaking. This also gives a possible mechanism for the annihilation of the symmetric component of the dark matter as well as a direct detection mechanism.

Dark matter masses in this range may give an explanation of the DAMA observations [13-16]. The DAMA experiment has observed an annual modulation with $8.2 \sigma$ significance consistent with WIMP scattering. Other direct detection experiments are sensitive to lower cross sections, but have higher energy thresholds, so a WIMP in the $10 \mathrm{GeV}$ mass range may explain the DAMA signal while still being consistent with other null results [16,17]. There remains some controversy, however, since the region consistent with the DAMA signal depends on the choice of binning [18]. The fit is particularly sensitive to the 2$2.5 \mathrm{keV}$ nuclear recoil bin; this bin taken by itself tends to shift the fit to larger dark matter masses [19]. We therefore emphasize that the models discussed here are interesting independently of the motivation from the DAMA observation.

The main features of our models are as follows.

(i) At a high temperature, a $B-L$ asymmetry is generated. Below this temperature $B-L$ is preserved, but new higher-dimension effective interactions that exchange the $B-L$ number between the standard model and dark matter are in thermal equilibrium.

(ii) The interactions that exchange the $B-L$ asymmetry decouple at lower temperatures, and a dark matter asymmetry is frozen in. There may still be couplings in equilibrium between the dark matter and the standard model that do not transfer the asymmetry.

(iii) At temperatures below the mass of the dark matter particle, particle-antiparticle annihilations eliminate the symmetric component of the dark matter density, leaving behind a relic density proportional to the particle-antiparticle asymmetry. These annihilations may occur either through the interactions that generate the dark matter mass or via the operator that transfers the asymmetry.

(iv) Direct detection of dark matter may occur, also through the interactions that generate the dark matter mass or via the operator that transfers the asymmetry.

(v) In supersymmetric models, the dark matter particle is naturally the lightest particle charged under a discrete $R$ symmetry, and the would-be lightest supersymmetric partner (LSP) decays into pairs of dark matter particles plus standard model particles. These decays may have a macroscopically displaced vertex.

This paper is organized as follows. In Sec. II, we describe concrete models and explain in detail how they give rise to the observed dark matter density. In Sec. III, we discuss direct detection signals. In Sec. IV, we discuss novel collider signals in this class of models. Section V contains our conclusions.

\section{MODELS}

It is simple to construct specific models that generate $\mathrm{ADM}$, and we will give three examples below. We find it simplest to explain the details of the mechanism in terms of a specific "reference" model. The remaining models will be described more briefly.

\section{A. Reference model: $L=\frac{1}{2} \mathrm{ADM}$}

We begin with a supersymmetric model in which the dark matter carries the lepton number. Supersymmetry (SUSY) is not necessary for the dark matter mechanism we are studying, but it allows a direct connection to a realistic and compelling model of electroweak physics and leads to very interesting collider phenomenology. Before going into the details of the model, we outline its general features:

(i) The dark matter sector consists of a pair of gauge singlet chiral superfields $X, \bar{X}$ with $L= \pm \frac{1}{2}$. This allows a supersymmetric mass term of the form $\bar{X} X$. There may be $\Delta L=2$ breaking of the lepton number from Majorana neutrino masses, but a $Z_{4}$ subgroup of $U(1)_{L}$ remains unbroken. This forbids Majorana mass terms of the form $X^{2}$ and $\bar{X}^{2}$ that can efficiently wipe out the asymmetry, and also guarantees that the lightest component of $X$ is a stable dark matter candidate.

(ii) A $B-L$ asymmetry generated at high scales is transferred to the dark matter via the effective interaction

$$
\Delta W_{\text {eff }}=\frac{1}{M_{i}} \bar{X}^{2} L_{i} H_{u},
$$

where $M_{i}$ is a high mass scale parametrizing the new physics that generates the interaction. The lowestdimension interactions allowed by the unbroken $Z_{4}$ subgroup of $U(1)_{L}$ are dimension-5 operators of the form $\Delta W \sim X^{4}$. As long as these drop out of equilibrium at a temperature where Eq. (2.1) is still in equilibrium, the asymmetry will be transferred to the visible sector. The interaction in Eq. (2.1) naturally goes out of equilibrium as the temperature drops further, and the dark matter asymmetry freezes in.

(iii) The dark matter mass is close to the electroweak scale, suggesting that it arises from electroweak symmetry breaking. This can occur in the next-tominimal supersymmetric standard model (NMSSM), where the $X$ mass arises from the coupling to a singlet $S$ that gets a vacuum expectation value (VEV) at the weak scale.

(iv) The annihilation of the symmetric component of the dark matter thermal density can occur through Higgs 
exchange in the NMSSM, or via the interaction that transfers the asymmetry.

(v) Direct detection can occur through the same interactions that are responsible for the annihilation. Either can give a signal in upcoming experiments.

(vi) The operator in Eq. (2.1) violates the usual $R$ parity, but preserves a $Z_{4} R$ symmetry under which the minimal supersymmetric standard model (MSSM) fields have the usual $R$ parity assignments of \pm 1 while

$$
X(\theta) \mapsto i X(-\theta), \quad \bar{X}(\theta) \mapsto-i \bar{X}(-\theta),
$$

where $\theta$ is the superspace coordinate. The would-be LSP can therefore decay into pairs of $X$ particles via the operator in Eq. (2.1). The $Z_{4} R$ symmetry forbids $R$-parity violating operators from being generated in the visible sector.

We now describe the model and the mechanism in more detail.

We begin by briefly discussing the UV completion of this model. The effective interaction in Eq. (2.1) can be obtained from a theory with a heavy pair of chiral multiplets $N, \bar{N}$ with $L= \pm 1$, i.e. vectorlike sterile neutrinos:

$$
\Delta W=M \bar{N} N+\lambda^{\prime} N \bar{X}^{2}+y_{i}^{\prime} \bar{N} L_{i} H_{u} .
$$

Another possibility is a vectorlike pair of electroweak doublets $D$ and $\bar{D}$ with $L= \pm \frac{1}{2}$ :

$$
\Delta W=M \bar{D} D+\lambda^{\prime} \bar{X} D H_{u}+y_{i}^{\prime} L_{i} \bar{D} \bar{X} .
$$

Either model generates Eq. (2.1) with $M_{i}=M /\left(\lambda^{\prime} y_{i}^{\prime}\right)$. Note that in the second model in Eq. (2.4), the Higgs VEV gives a mixing between $X$ and the neutral component of $D$; this can be treated as a small perturbation as long as $M \gg \lambda^{\prime} v_{u}$. The second model is more natural if Majorana neutrino masses are generated from a standard seesaw mechanism. The reason is that the sterile neutrino Majorana mass in the standard seesaw naturally arises from the VEV of a field with $L=2$. Such a field has no renormalizable couplings to the fields in Eq. (2.4), and therefore the lepton number can be an accidental symmetry in the dark matter sector. This also allows the $B-L$ asymmetry to be generated by standard leptogenesis.

We now discuss the generation of the dark matter asymmetry in this model. In the early Universe, nonrenormalizable effective interactions such as Eq. (2.1) give rise to interactions whose rate drops faster than the expansion rate as the temperature of the Universe drops. It is therefore natural for this operator to have been in equilibrium in the early Universe, but to be out of equilibrium today. This is exactly what is required to distribute a $B-L$ asymmetry in the early Universe to the $X$ fields. This goes out of equilibrium for $T \gtrsim 100 \mathrm{GeV}$ provided that $M \geq 10^{9} \mathrm{GeV}$. For this coupling strength, the bounds on induced lepton flavor violation such as $\mu \rightarrow e \gamma$ are well below the experimental limits.
It is also possible for the interactions that transfer the asymmetry to go out of equilibrium at temperatures below the electroweak scale but above the dark matter mass even if $M \ll 10^{9} \mathrm{GeV}$. This is a small temperature range (roughly 10 to $100 \mathrm{GeV}$ ), but we will see this arises naturally for a wide range of parameters. The component interactions arising from Eq. (2.1) contain at most two fermion fields, so the only interactions that change the $X$ fermion number arising directly from Eq. (2.1) involve sneutrino and/or Higgs particles, e.g. $\tilde{\nu} \leftrightarrow \bar{X} \bar{X}$. These interactions become ineffective at temperatures below the sneutrino mass because of the exponentially small abundance of sneutrinos. The rate is

$$
\Gamma(\tilde{\nu} \leftrightarrow X X) \sim \frac{n_{\tilde{\nu}}}{n_{X}} \frac{1}{16 \pi}\left(\frac{v_{u}}{M}\right)^{2} m_{\tilde{\nu}} .
$$

This freezes out when the rate drops below the Hubble expansion rate, which occurs for $T \lesssim m_{\tilde{\nu}} / 40$ for $M \sim$ $\mathrm{TeV}$. In addition, there are transitions between light particles generated by integrating out virtual heavy particles. Since all light particles are neutral under the $Z_{4} R$ symmetry, we need two insertions of the operator in Eq. (2.1). The leading contribution arises from integrating out virtual sneutrinos and neutralinos, and gives rise to an effective operator

$$
\mathcal{L}_{\text {eff }} \sim \frac{v_{u}^{2}}{M^{2} m_{\tilde{\nu}}^{4} m_{\tilde{B}}}(\bar{X} \bar{X})^{2} \nu \nu
$$

The asymmetry-transferring processes mediated by this interaction (e.g. $\bar{X} \bar{X} \leftrightarrow X X \bar{\nu} \bar{\nu}$ ) have a rate that falls rapidly as the Universe cools,

$$
\Gamma \sim \frac{1}{16 \pi}\left(\frac{1}{8 \pi^{2}}\right)^{2}\left(\frac{v_{u}^{2}}{M^{2} m_{\tilde{\nu}}^{4} m_{\tilde{B}}}\right)^{2} T^{11},
$$

where the prefactor is an estimate of 4-body phase space. This goes out of equilibrium for

$$
T \lesssim 20 \mathrm{GeV}\left(\frac{M}{\mathrm{TeV}}\right)^{4 / 9}\left(\frac{m}{100 \mathrm{GeV}}\right)^{10 / 9},
$$

where $m \sim m_{\tilde{\nu}} \sim m_{\tilde{B}}$. We see that for $m_{X} \sim 10 \mathrm{GeV}$, the temperature where the interactions decouple can be above $m_{X}$ even if $M$ is near the weak scale. In this case, the interactions in Eq. (2.6) fall out of equilibrium before the dark matter becomes nonrelativistic, and the dark matter asymmetry is not washed out. ${ }^{1}$

For low values of $M$, bounds from lepton flavor violation such as $\mu \rightarrow e \gamma$ can be satisfied if the coupling in Eq. (2.1) is dominant to third generation leptons. This is what we

\footnotetext{
${ }^{1}$ Sphaleron transitions may fall out of equilibrium in this temperature range, but this does not have a large effect on the dark matter asymmetry. Below the sphaleron decoupling temperature $B$ and $L$ are effectively separately conserved, but this does not prevent the operator in Eq. (2.1) from transferring the asymmetry.
} 
expect if flavor symmetry is most badly broken for the heavier generations. For these low-scale models, the interaction in Eq. (2.1) may provide both an annihilation and a direct detection mechanism, which will be discussed in Sec. III below.

We now discuss the calculation of the $X$ particleantiparticle asymmetry. Because the asymmetry is transferred by interactions in equilibrium, we can compute the $X$ asymmetry in terms of the particle-antiparticle asymmetries of the standard model using standard equilibrium methods [20]. The value of the $X$ asymmetry at low temperatures depends on the temperature where the interactions in Eq. (2.1) drop out of equilibrium. We first discuss the case where these interactions drop out of equilibrium above the electroweak phase transition. We then have

$$
X=-\frac{11}{79}(B-L) \text {, }
$$

where $X$ is the " $X$ number" charge. $B-L$ is preserved by the electroweak phase transition, so the present baryon asymmetry is proportional to $B-L .^{2}$ Sphaleron transitions that violate $B$ and $L$ are in equilibrium below the electroweak phase transition [21]. The precise relation between $B$ and $B-L$ depends on finite mass effects, e.g. for the top quark. Numerically, however, these do not make a large difference, and we find

$$
\frac{B}{B-L} \simeq 0.31 \text {. }
$$

Assuming the $X$ asymmetry is responsible for the observed dark matter density results in a prediction for the mass of the $X$ particle if the interactions fall out of equilibrium above the electroweak phase transition:

$$
m_{X} \simeq 2.4 \mathrm{GeV} \frac{\Omega_{\mathrm{DM}}}{\Omega_{B}} \simeq 11 \mathrm{GeV} .
$$

The fact that the $X$ mass is somewhat larger than the naïve estimate of $5 \mathrm{GeV}$ is due to $X<B$, which in turn can be traced to the fact that the model contains more baryons than $X$ particles: in relativistic equilibrium conserved charges are proportional to the number of degrees of freedom carrying that charge. ${ }^{3}$

It is also possible that the interactions in Eq. (2.1) decouple below the electroweak phase transition. In this case, integrating out both the top and the superpartners, we obtain

$$
\frac{X}{B}=\frac{13}{40},
$$

and therefore

\footnotetext{
${ }^{2} \mathrm{We}$ are assuming that there is no significant baryon asymmetry generated during the electroweak phase transition.

${ }^{3}$ We must also impose the condition that the Universe has no net electric charge. Since $X$ does not carry charge, this condition restricts only the relative number of standard model particles, and does not affect the scaling argument above.
}

$$
m_{X} \simeq 13 \mathrm{GeV} .
$$

We now discuss the origin of the dark matter mass. This is a supersymmetric Dirac mass arising from a superpotential term $\Delta W=m_{X} \bar{X} X$. The question of why $m_{X}$ is close to the weak scale is similar to the " $\mu$ problem" of supersymmetric models, which is explaining the origin of the supersymmetric Higgs mass term $\Delta W_{\text {eff }}=\mu H_{u} H_{d}$. Perhaps the simplest solution is the NMSSM in which the required mass terms are given by the VEV of a singlet field $S$ :

$$
\Delta W=\lambda_{X} S X \bar{X}+\lambda_{H} S H_{u} H_{d}+\frac{\kappa}{3} S^{3} .
$$

This model naturally generates a VEV for $S$ of the order of the electroweak scale and gives the required mass terms for Higgs and $X$ particles. Very important for dark matter phenomenology, it also gives a direct coupling of $X$ to the standard model, allowing the dark matter to be directly detected.

The final ingredient is that the thermal abundance of $X$ particles and antiparticles must efficiently annihilate, so that the relic density of dark matter is given by the $X$ particle-antiparticle asymmetry. This requires $\left\langle\sigma_{\text {ann }} v\right\rangle \gtrsim$ $\mathrm{pb}$. In the context of the NMSSM, a simple possibility is $\bar{X} X \rightarrow a a$, where $a$ is the lightest pseudoscalar in the Higgs sector. This is unsuppressed in the early Universe as long as $m_{a} \lesssim m_{X}$. It is natural for $a$ to be light if $A$ terms are small, in which case $a$ is a pseudo-Nambu-Goldstone boson of a global $U(1)_{R}$ symmetry. The annihiation comes from the coupling,

$$
\Delta \mathcal{L}_{\text {eff }}=m_{X} \bar{X} X e^{i a / s}+\text { H.c., }
$$

where $s / \sqrt{2}=\langle S\rangle$, which gives an annihilation cross section

$$
\left\langle\sigma v_{\text {rel }}\right\rangle=\frac{1}{16 \pi} \frac{m_{X}^{2}}{s^{4}} .
$$

This is larger than $1 \mathrm{pb}$ for $s<200 \mathrm{GeV}$. This requires superpotential couplings $\lambda, \kappa \sim \mathcal{O}(1)$ to generate the correct spectrum in the NMSSM, and the resulting theory is not perturbative up to the grand unified theory scale. This means that an extension of the Higgs sector is required at high scales, such as in "fat" Higgs models [22]. The pseudoscalar $a$ can decay promptly to $\bar{b} b$ (for $m_{a} \gtrsim$ $10 \mathrm{GeV}$ ) or $\tau^{+} \tau^{-}$(for $2 \mathrm{GeV} \lesssim m_{a} \lesssim 10 \mathrm{GeV}$ ), so there are no further cosmological consequences. Interestingly, this model points to the same region of NMSSM parameter space where nonstandard Higgs decays such as $h^{0} \rightarrow a a$ followed by $a \rightarrow \bar{b} b$ or $\tau^{+} \tau^{-}$can dominate, which may alleviate the naturalness problems of supersymmetry [23]. We will also see that this model may give rise to a direct detection signal.

Another possibility for annihilation is that the singlet couples weakly to the Higgs fields. In this case the $\mu$ term is not explained by the VEV of $S$; it may arise e.g. by the 
Giudice-Masiero mechanism [24]. The theory can be perturbative up to the grand unified theory scale without additional structure. The light $a$ can result from an approximate $U(1)_{R}$ symmetry acting only on $S$, and the decay $a \rightarrow \bar{b} b$ or $\tau^{+} \tau^{-}$need only to be faster than a second to avoid constraints from nucleosynthesis. This annihilation mechanism does not give any observable direct detection or collider signals.

Another possibility for annihilation arises from the fields in the UV completion Eq. (2.4) if the doublets $D$ and $\bar{D}$ are light. Assuming dominance of heavy flavors, we then have the annihilation channels $\bar{X} X \rightarrow \bar{\nu} \nu, \tau^{+} \tau^{-}$from the $t$-channel exchange of the scalar component of the doublet with rate

$$
\left\langle\sigma v_{\text {rel }}\right\rangle=\frac{1}{16 \pi} \frac{y^{14} m_{X}^{2}}{m_{\tilde{D}}^{4}}
$$

This is larger than a pb for $m_{\tilde{D}} / y^{\prime}<190 \mathrm{GeV}$. The coupling $y^{\prime}$ breaks lepton flavor symmetry, and suppressing lepton flavor-violating processes such as $\mu \rightarrow e \gamma$ requires nontrivial structure in the lepton flavor sector. For example, there may be an approximate $U(1)_{L}^{3}$ forbidding lepton mixing that is broken only by small neutrino masses. The charged doublet scalar can be pair produced and decays to $\tau^{ \pm}+\mathbb{E}_{T}$. LEP bounds give $m_{\tilde{D}^{ \pm}}>92 \mathrm{GeV}$ [25]; there is currently no Tevatron search for this mode. In order for the operator in Eq. (2.1) that transfers the asymmetry to decouple at temperatures above $m_{X}$, we need $\lambda^{\prime} \ll 1$ in Eq. (2.4). Note that this also suppresses the mixing between $X$ and the neutral component of the doublet, which would otherwise lead to coupling of $X$ to the $Z$. Having $\lambda^{\prime} \ll 1$ is natural, because in the limit $\lambda^{\prime} \rightarrow 0$ there is an enhanced global $U(1)$ symmetry under which $X$ and $D$ have opposite charges. As we will discuss in Sec. III, this model has a direct detection cross section via a charge radius interaction that is near the experimental limit, making this version of the mode phenomenologically very interesting.

There are other possibilities for the annihilation, such as annihilation into light hidden sector fields or other couplings to standard model fields, e.g. via a $Z^{\prime}$. Another interesting possibility is to explore models where annihilation occurs via new strong dynamics, as in "hidden valley" [26] or "quirk" [27] models. We leave these possibilities for future work.

\section{B. $B=\frac{1}{2} \mathrm{ADM}$}

We now describe a supersymmetric model in which the dark matter carries the baryon number. The model is a very simple variation of the previous model, so our discussion will be very brief. The model consists of the MSSM plus a pair of gauge singlet chiral superfields $X, \bar{X}$ with $B= \pm \frac{1}{2}$. The lowest-dimension operator that can transfer the baryon asymmetry to $X$ is

$$
\Delta W_{\text {eff }}=\frac{1}{M_{i j k}^{2}} \bar{X}^{2} u_{i} d_{j} d_{k}
$$

If this interaction goes out of equilibrium above the electroweak phase transition, we find

$$
X=-\frac{11}{79}(B-L) .
$$

Amusingly, this is precisely the same result as in the previous model, and we again find

$$
m_{X} \simeq 11 \mathrm{GeV}
$$

if the interaction in Eq. (2.18) decouples above the electroweak phase transition. The $\bar{X} X$ annihilation and the generation of the $X$ mass are very similar to the previous model, and we will not repeat the discussion. A significant difference between this model and the $L=\frac{1}{2}$ model is the long lifetime of the LSP due to the high dimension of the transfer operator. As we will see in Sec. IV below, the scale $M$ in some cases must be of the order of a TeV or smaller in order to avoid decays on cosmological time scales.

\section{C. $L=1$ (sterile neutrino) ADM}

We now consider a model in which the dark matter has $L=1$, like a sterile neutrino. The lowest-dimension coupling to the standard model that can transfer the lepton asymmetry to $X$ is then

$$
\Delta \mathcal{L}_{\text {eff }}=\frac{1}{M_{i j}^{4}} \bar{X}^{2}\left(L_{i} H\right)\left(L_{j} H\right)+\text { H.c. }
$$

Majorana neturino masses conventionally arise from an effective operator of the form $(L H)^{2}$, so any model that generates the interaction in Eq. (2.21) and Majorana masses necessarily generates a Majorana mass term for $X$ at some level. This will efficiently wipe out any $X$ asymmetry, so this model is most natural with Dirac neutrino masses.

If the interaction in Eq. (2.21) goes out of equilibrium above the weak scale, we have

$$
X=-\frac{12}{49}(B-L),
$$

corresponding to a dark matter particle mass $m_{X} \simeq 6 \mathrm{GeV}$.

A UV completion of Eq. (2.21) can be obtained by adding an additional scalar Higgs doublet $H^{\prime}$ with couplings

$$
\Delta \mathcal{L}=y_{i}^{\prime} L_{i} H^{\prime} \bar{X}-\frac{\lambda^{\prime}}{4}\left[\left(H^{\dagger} H^{\prime}\right)^{2}+\text { H.c. }\right]+\cdots .
$$

Integrating out $H^{\prime}$ generates Eq. (2.21) with $1 / M_{i j}^{4} \sim$ $\lambda^{\prime} y_{i}^{\prime} y_{j}^{\prime} / m_{H^{\prime}}^{4}$. Note that $H^{\prime}$ is odd under the $Z_{2}$ symmetry that prevents $X$ decay, so we must assume that $\left\langle H^{\prime}\right\rangle=0$.

Exchange of $H^{\prime}$ can give rise to annihilation for the symmetric component of the $X$ relic density if $m_{H^{\prime}} / y^{\prime} \lesssim$ $200 \mathrm{GeV}$. This is very similar to exchange of the doublet scalars in the $L=\frac{1}{2}$ model. It requires $\lambda^{\prime} \ll 1$ in order to 
decouple the transfer of the asymmetry above $m_{X}$, and nontrivial lepton flavor structure to avoid lepton flavor violation such as $\mu \rightarrow e \gamma$. Direct detection signals from the light $H^{\prime}$ will be discussed in Sec. IV below.

This model relies on the existence of light scalars $\left(H^{\prime}\right.$ and the Higgs), and so must be embedded in a framework that makes such scalars natural. To embed this model into a supersymmetric model, we add two additional "Higgs" chiral multiplets $H_{u, d}^{\prime}$, as well as $S U(2)_{W}$ triplets $\Delta_{u, d}$ with $Y=\mp 1$. The relevant terms in the superpotential are

$$
\begin{aligned}
\Delta W \sim & L H_{u}^{\prime} \bar{X}+\Delta_{u}\left(H_{u}^{2}+H_{u}^{\prime 2}\right)+\Delta_{d}\left(H_{d}^{2}+H_{d}^{\prime 2}\right) \\
& +\bar{X} X+H_{u} H_{d}+H_{u}^{\prime} H_{d}^{\prime}+\Delta_{u} \Delta_{d} .
\end{aligned}
$$

Integrating out $\Delta_{u, d}$ then generates Eq. (2.23). The mass terms may arise from the VEV of a singlet, as discussed previously. These interactions preserve both an $R$ parity under which the $X$ fermion is even, and a $Z_{2}$ symmetry under which $X, \bar{X}$, and $H_{u, d}^{\prime}$ are odd and all other fields are even. It is therefore possible that this model contains a stable LSP in addition to the dark matter particle. In this case, the relic density of the LSP is constrained to be less than the observed dark matter density. Alternatively, one of the $Z_{2}$ symmetries can be broken by interactions such as $\Delta W \sim X e^{c}\left(H_{u} H_{d}\right)\left(H_{u}^{\prime} H_{d}\right)$, allowing the LSP to decay.

\section{DIRECT DETECTION}

Asymmetric dark matter requires only very weak interactions with the visible sector to explain the dark matter asymmetry, and so there is no guarantee of an observable direct detection cross section. However, the symmetric component of the dark matter relic density must be efficiently annihilated away, and if this annihilation goes into standard model fields, the interactions responsible for the annihilation can give rise to direct detection similar to WIMP dark matter.

In the previous section we presented two different minimal possibilities for the interaction that annihilates away the symmetric part of the dark matter, and each of them leads to a different possible direct detection mechanism.

(i) The annihilation may go through a singlet Higgs field whose VEV gives the dark matter mass. In this case the mixing between the singlet and the doublet Higgs fields couples the dark matter to nucleons, giving a potential direct detection signal several orders of magnitude below current bounds.

(ii) The annihilation may proceed via $t$-channel exchange of a doublet scalar at the weak scale that is part of the UV completion of the interaction that transfers the asymmetry. In this case, there is a magnetic moment coupling of the dark matter to nucleons that is closer to current bounds.

We now discuss these possibilities in turn.

We first discuss singlet Higgs exchange. The coupling in Eq. (2.14) gives a coupling of the lightest scalar Higgs

$$
g_{\bar{X} X h^{0}} \sim \lambda_{X} \sin \theta,
$$

where $\theta$ is a Higgs-singlet mixing angle. For a standard model Higgs coupling to the nucleon, this gives a spinindependent dark matter-nucleon cross section

$$
\begin{aligned}
\sigma_{\exp }(X n \rightarrow X n) & =\frac{1}{A^{2}} \frac{m_{X n}^{2}}{m_{X N}^{2}} \sigma(X N \rightarrow X N) \\
& \simeq 5 \times 10^{-43} \mathrm{~cm}^{2} \times g_{\bar{X} X h^{0}}^{2}\left(\frac{m_{h^{0}}}{100 \mathrm{GeV}}\right)^{-4} .
\end{aligned}
$$

Here $\sigma_{\exp }$ is the experimentally quoted dark matternucleon cross section and $m_{x y}$ is the reduced mass (see e.g. Ref. [1]). The best bound on this cross section for $m_{X} \simeq$ $15 \mathrm{GeV}$ comes from XENON [28], $\sigma_{\text {exp }} \lesssim 9 \times$ $10^{-44} \mathrm{~cm}^{2}$, with a similar bound from CDMS [29]. Since $\lambda_{X}=\sqrt{2} m_{X} / s \sim 0.1$ for $s \sim v$ near the weak scale, the direct detection rate is about 2 orders of magnitude below current sensitivity in this mass range.

In this model, the lightest pseudoscalar $a$ is light, and its exchange gives rise to an effective coupling of dark matter to quarks,

$$
\begin{aligned}
\Delta \mathcal{L}_{\mathrm{eff}}= & \frac{m_{X}}{s m_{a}^{2}} \bar{X} i \gamma_{5} X\left[\sum_{u} \frac{m_{u} \cot \beta}{v} \bar{u} i \gamma_{5} u\right. \\
& \left.+\sum_{d} \frac{m_{d} \tan \beta}{v} \bar{d} i \gamma_{5} d\right] .
\end{aligned}
$$

This gives a spin-dependent coupling to nucleons that is well below current experimental bounds.

We now turn to direct detection signals from the interaction that transfers the asymmetry. For the $L=\frac{1}{2}$ and $L=$ 1 model, the minimal UV completion involves a scalar doublet, and if it is light it can give sufficient annihilation. ${ }^{4}$ In this case, there is a one-loop magnetic moment and charge radius coupling that can contribute to direct detection. (For a recent discussion of dark matter detection through the magnetic moment, see [30].) The magnetic moment and charge radius (defined in terms of the standard electromagnetic form factors by $F_{1}\left(q^{2}\right)=q^{2} r^{2} / 6+\cdots$, $\left.F_{2}\left(q^{2}\right)=\mu / 2 m_{X}+\cdots\right)$ were computed in Ref. [31]:

$$
\begin{gathered}
\mu=\frac{y^{\prime 2}}{32 \pi^{2}} \frac{m_{X}^{2}}{m_{\phi^{ \pm}}^{2}}, \\
r^{2}=-\frac{y^{\prime 2}}{288 \pi^{2}} \frac{1}{m_{\phi^{ \pm}}^{2}}\left[\ln \frac{m_{\phi^{ \pm}}}{m_{\tau}}-\frac{3}{4}\right],
\end{gathered}
$$

where $\phi^{ \pm}$is the charged scalar in the doublet, and we use their definitions of $\mu$ and $r^{2}$. The charge radius contribu-

\footnotetext{
${ }^{4}$ The UV completion of the $B=\frac{1}{2}$ model also involves electrically charged fields that may be light and lead to annihilation and direct detection. We will not discuss this possibility here.
} 
tion to the cross section is IR divergent. This is regulated using the "energy transfer" nuclear cross section, which is given by [31]

$$
\begin{aligned}
\sigma(X N \rightarrow X N)= & \frac{\pi Z^{2} \alpha^{2}}{m_{X}^{2}}\left[\mu^{2}+\left(\frac{m_{N}}{m_{N}+m_{X}}\right)^{2}\right. \\
& \left.\times\left(24 r^{2} m_{X}^{2}-\mu\right)^{2}\right],
\end{aligned}
$$

where $m_{N}$ is the nucleus mass. The cross section is numerically dominated by the charge radius term. Note that $\sigma \propto m_{X}^{2}$, so that $\sigma_{n}^{\exp }$ is independent of $m_{X}$ for $m_{X} \ll m_{N}$. We obtain

$$
\sigma_{n}^{\exp } \simeq 8 \times 10^{-44} \mathrm{~cm}^{2}\left(\frac{Z / A}{0.4}\right)^{2}\left(\frac{m_{\phi^{ \pm}} / y^{\prime}}{100 \mathrm{GeV}}\right)^{-4} .
$$

This is below current bounds, but may give a signal in upcoming experiments.

Finally, we mention that it is also possible to have direct detection from interactions that are not directly motivated by the physics of generating the dark matter asymmetry. For example, a $Z^{\prime}$ mediator can also produce spinindependent cross sections of this size,

$$
\sigma_{n}^{\exp } \simeq 10^{-41} \mathrm{~cm}^{2}\left(\frac{g_{\bar{X} X Z^{\prime}} g_{\bar{u} u Z^{\prime}}}{10^{-1}}\right)^{2}\left(\frac{1 \mathrm{TeV}}{M_{Z^{\prime}}}\right)^{4} .
$$

This corresponds to a WIMP mass detected by DAMA of $\approx 7 \mathrm{GeV}$ [16-18], which results naturally from the models discussed above.

\section{COLLIDER SIGNALS}

The new sector in ADM includes new states at the weak scale and below, and thus have the potential to affect collider phenomenology. The most significant possibilities are:

(i) New Higgs boson decays, both invisible and into lighter decaying scalars.

(ii) Supersymmetry with NLSP decay resulting in reduced missing energy, more leptons, and/or new displaced vertices. Kinematic shapes in cascade decays will also differ from standard scenarios.

(iii) New charged states at the weak scale and/or colored states at a $\mathrm{TeV}$.

These signals can coexist in a single model. We now discuss them in turn.

We begin with Higgs phenomenology. If the NMSSM explains the dark matter annihilation, then the Higgs has phenomenologically interesting couplings to both the dark matter and the light pseudoscalar into which the dark matter annihilates. The couplings $\lambda_{H}$ and $\kappa$ must be $\mathcal{O}(1)$ to allow for efficient enough annihilation. The mixing angle between the lightest $C P$ even Higgs and the scalar singlet $s$ is

$$
\sin \theta_{s h^{0}} \simeq \frac{2 \mu v}{s^{2}} \sin 2 \beta \sim \sin 2 \beta,
$$

where $\mu$ is the effective mu term, and the last approximation is due to annihilation requirements and chargino bounds. Thus, the Higgs can decay to $X \bar{X}$ by mixing with $s$ with a decay width

$$
\frac{\Gamma(h \rightarrow X \bar{X})}{\Gamma(h \rightarrow b \bar{b})} \simeq \frac{\lambda_{X}^{2} \sin ^{2} 2 \beta v^{2}}{3 m_{b}^{2}} .
$$

With $\lambda_{X} \sim 0.1$ to produce the correct $X$ mass and using the value of $m_{b}$ at the electroweak scale, we find that this new decay is competitive at moderate $\tan \beta$, implying a large invisible width for the Higgs.

In addition, the Higgs can decay into pairs of light pseudoscalars in this model. This can dominate the Higgs width, especially since the couplings $\lambda_{H}$ and $\kappa$ are $\mathcal{O}(1)$. The light pseudoscalar mixes with the heavy $C P$-odd Higgs $A^{0}$ and decays into $b \bar{b}$ or $\tau \bar{\tau}$, thus producing a dominant decay of $h \rightarrow 4 b$ or $h \rightarrow 4 \tau$ (for a review, see [32]). Of course a large partial width into these modes will suppress the invisible decay mode discussed above.

We now turn to the decay of the would-be LSP in supersymmetric theories. This occurs in the $L=\frac{1}{2}$ and $B=$ $\frac{1}{2}$ models described above.

There are a large number of possible decay modes depending on the model and the identity of the LSP. These have certain common features that we point out before discussing the individual cases. As discussed above, in these models the usual LSP is not the lightest particle charged under a discrete $R$ symmetry. Instead, there is a discrete $Z_{4}$ symmetry that allows the would-be LSP (NLSP) to decay to pairs of dark matter particles. (We will refer to the would-be LSP as the NLSP.) This means that LSP-mass reconstruction techniques [33] must be generalized to determine the mass of the dark matter. Also, the decays are suppressed both by the scale $M$ in the higher-dimension operators Eqs. (2.1) and (2.18) and by the fact that the decays are often many-body decays. This leads to the possibility of displaced vertices in the decays. These operators have a nontrivial flavor structure, and considerations of approximate flavor symmetry suggest that they are largest for heavy flavors; if so, this leads to LSP decays involving heavy flavors, which may be tagged. The collider physics of these models is therefore extremely rich and interesting. In this paper, we will give only a sample of possible dominant LSP decays, leaving detailed investigation for future work.

We begin with the $L=\frac{1}{2}$ model. There are various possibilities for the identity of the NLSP. If the NLSP is a neutralino, it has the decay $\chi^{0} \rightarrow \nu \bar{X} \bar{X}$ or $\bar{\nu} X X$ via a virtual sneutrino. This is unfortunately completely invisible, and so gives no modification of standard LSP phenomenology even for macroscopic decay length. It is important to note, however, that the reconstructed LSP is 
not the dark matter particle in this case. If the neutralino is sufficiently heavy, there are also the 4-body visible decays $\chi^{0} \rightarrow h^{0} \nu \bar{X} \bar{X}, h^{0} \bar{\nu} X X, h^{+} \ell^{-} \bar{X} \bar{X}$, and $h^{-} \ell^{+} X X$. These decays proceed through a virtual left-handed slepton like the dominant decay mode, so the branching ratio is

$$
\mathrm{BR}\left(\chi^{0} \rightarrow h^{0} \nu \bar{X} \bar{X}\right) \sim \frac{1}{8 \pi^{2}}\left(\frac{m_{\chi^{0}}}{v_{u}}\right)^{2} .
$$

This can easily be $\sim 1 \%$ or larger and provide a window into LSP decay in this model. The decay length for the visible decays is

$$
\begin{aligned}
c \tau\left(\chi^{0} \rightarrow h^{0} \nu \bar{X} \bar{X}\right) \sim & \mathrm{mm}\left(\frac{M}{10^{6} \mathrm{GeV}}\right)^{2}\left(\frac{m_{\tilde{\nu}}}{200 \mathrm{GeV}}\right)^{4} \\
& \times\left(\frac{m_{\chi^{0}}}{100 \mathrm{GeV}}\right)^{-7} .
\end{aligned}
$$

We see that the decay vertex is displaced for $M \gtrsim$ $10^{4} \mathrm{GeV}$.

If the $X$ scalars are lighter than the MSSM neutralino, we have the decays $\chi^{0} \rightarrow \nu \tilde{X} \tilde{X}, \bar{\nu} \tilde{X} \bar{X}$. These proceed directly through the interaction in Eq. (2.1) without a virtual intermediate state and are therefore enhanced compared to $\chi^{0} \rightarrow \nu \bar{X} \bar{X}$. The decay length is

$$
c \tau\left(\chi^{0} \rightarrow \bar{\nu} \tilde{X} \tilde{X}\right) \sim \operatorname{cm}\left(\frac{M}{10^{8} \mathrm{GeV}}\right)^{2}\left(\frac{m_{\chi^{0}}}{100 \mathrm{GeV}}\right)^{-3},
$$

The $X$ scalars subsequently decay via $\tilde{X} \rightarrow \bar{X} \nu$, which is a completely invisible mode, or the subleading visible decay mode $\tilde{X} \rightarrow X \nu h^{0}, \tilde{X} \rightarrow X \ell^{ \pm} h^{\mp}$, assuming it is kinematically available. The branching ratio for the visible mode is suppressed only by 3-body phase space, and so it can have a branching ratio of up to $\sim 1 \%$ if the 3-body channel is fully open. The decay length for the visible mode is approximately the same as Eq. (4.5). The general pattern for $\chi^{0}$ decays is therefore qualitatively the same whether the scalar decay channel is open or not: the dominant decay is invisible, possibly with rare decays to Higgs plus missing energy, or charged Higgs plus charged lepton and missing energy.

We now consider the case where the NLSP is a slepton or squark. In this case, the dominant decay is through a virtual gaugino: $\tilde{q} \rightarrow q \tilde{\chi}^{*}$ or $\tilde{\ell} \rightarrow \ell \tilde{\chi}^{*}$. The virtual gaugino then decays through the same modes as given for the real gaugino above.

For example, suppose the NLSP is a right-handed stau. (We expect the heaviest flavor slepton or squark to be the lightest, since the Yukawa couplings drive down the scalar masses in the renormalization group equation.) This can occur, for example, in models of gauge-mediated SUSY breaking. If the $X$ scalars are heavy, the dominant decay mode is $\tilde{\tau}_{R} \rightarrow \tau \nu \bar{X} \bar{X}, \tau \bar{\nu} X X$ via a virtual neutralino. The decay length is

$$
\begin{aligned}
c \tau\left(\tilde{\tau}_{R} \rightarrow \tau \nu \bar{X} \bar{X}\right) \sim & \mathrm{mm}\left(\frac{M}{10^{6} \mathrm{GeV}}\right)^{2}\left(\frac{m}{200 \mathrm{GeV}}\right)^{6} \\
& \times\left(\frac{m_{\tilde{\tau}}}{100 \mathrm{GeV}}\right)^{-7},
\end{aligned}
$$

where we have assumed a common mass scale $m \sim m_{\tilde{\nu}} \sim$ $m_{\chi^{0}}$. The chargino couples to the right-handed stau only through the tau Yukawa, but this may be important at large $\tan \beta$. Virtual chargino exchange gives the decays

$$
\mathrm{BR}\left(\tilde{\tau}_{R}^{-} \rightarrow \nu_{\tau} \ell^{-} \bar{X} \bar{X}\right) \sim 10^{-4} \tan ^{2} \beta\left(\frac{m_{\chi^{0}}}{m_{\chi^{ \pm}}}\right)^{2},
$$

which may involve light leptons, depending on the flavor structure of the interaction in Eq. (2.1).

As for the neutralino NSLP, decays to $X$ scalars are dominant if they are kinematically allowed. The decays are $\tilde{\tau}_{R}^{-} \rightarrow \ell \nu \tilde{X} \tilde{X}$ with decay length

$$
\begin{aligned}
c \tau\left(\tilde{\tau}_{R} \rightarrow \ell \nu \tilde{X} \tilde{X}\right) \sim & \mathrm{cm}\left(\frac{M}{10^{7} \mathrm{GeV}}\right)^{2}\left(\frac{m}{200 \mathrm{GeV}}\right)^{2} \\
& \times\left(\frac{m_{\tilde{\tau}}}{100 \mathrm{GeV}}\right)^{-5} .
\end{aligned}
$$

This is followed by $\tilde{X} \rightarrow \bar{X} \nu$ with decay length given by Eq. (4.5).

The right-handed stau will mix with the left-handed stau via $A$ terms and the $\mu$ term times the tau Yukawa coupling. It is also possible that the left-handed stau is the LSP. We therefore consider the decays of a left-handed stau. If kinematically available, the most important decay is directly through the operator in Eq. (2.1), $\tilde{\tau}_{L} \rightarrow h^{-} X X$. The decay length is given by Eq. (4.5). If this channel is not open, the leading decay is $\tilde{\tau}_{L} \rightarrow W X X$ via a virtual snuetrino. The decay rate is parametrically the same as Eq. (4.6) . For sufficiently large $m_{\tilde{\tau}}$, we also have the decay $\tilde{\tau}_{L}^{-} \rightarrow$ $X X \bar{t} b$ and $\tilde{\tau}_{L}^{+} \rightarrow \bar{X} \bar{X} \bar{b} t$. The ratio of decay rates is

$$
\frac{\Gamma\left(\tilde{\tau}_{L}^{-} \rightarrow X X \bar{t} b\right)}{\Gamma\left(\tilde{\tau}_{L}^{-} \rightarrow W^{-} X X\right)} \sim 0.1\left(\frac{m_{\tilde{\nu}}}{m_{H^{ \pm}}}\right)^{4}\left(\frac{m_{\tilde{\tau}}}{300 \mathrm{GeV}}\right)^{2} .
$$

This may be the dominant decay, depending on the superpartner masses.

Returning to the case of right-handed stau LSP, the mixing with $\tilde{\tau}_{L}$ allows the decay to the final states discussed for $\tilde{\tau}_{L}$ decay above:

$$
\Delta \Gamma\left(\tilde{\tau}_{R} \rightarrow \cdots\right) \sim\left(\frac{m_{\tau} \mu \tan \beta}{m^{2}}\right)^{2} \Gamma\left(\tilde{\tau}_{L} \rightarrow \cdots\right) .
$$

Therefore, $\tilde{\tau}_{R} \rightarrow h^{-} X X$ may be an important decay mode in some models, particularly at large $\tan \beta$.

If a squark (e.g. the stop) is the LSP, then the dominant decay modes are $\tilde{q} \rightarrow q \nu \bar{X} \bar{X}, q \bar{\nu} X X$ via a virtual neutralino, or $\tilde{q} \rightarrow q \ell \bar{X} \bar{X}, q \bar{\ell} X X$ via a virtual chargino. The latter decay will be suppressed if the LSP is dominantly a right-handed squark. The decay length is as in Eq. (4.6) with the obvious replacements. 
We now turn to the $B=\frac{1}{2}$ model. In general, the decay lengths in the $B=\frac{1}{2}$ model are longer than the $L=\frac{1}{2}$ model, because the operator in Eq. (2.18) is suppressed by a higher power of $M$. In addition, some decays are loop suppressed. We begin with the case of neutralino NSLP. If the $X$ scalars are heavy, the neutralino will decay via $\chi^{0} \rightarrow$ $u d d \bar{X} \bar{X}$ or $\bar{u} \bar{d} \bar{d} X X$. The diagram involves a virtual quark connecting to $X$ fermions, with the remaining squarks converting to quarks via a loop. The decay length for this process is

$$
\begin{aligned}
c \tau\left(\chi^{0} \rightarrow X X q q q\right) \sim & 100 \mathrm{~m}\left(\frac{M}{\mathrm{TeV}}\right)^{4}\left(\frac{m}{500 \mathrm{GeV}}\right)^{6} \\
& \times\left(\frac{m_{\chi^{0}}}{100 \mathrm{GeV}}\right)^{-11} .
\end{aligned}
$$

This estimate is highly uncertain due to the estimate of 4body phase space and the loop factor. For such large displaced vertices the collider physics of the SUSY LSP is unchanged, but the LSP reconstructed at colliders is not the dark matter. As above, decay to the $X$ scalars is the preferred mode when it is kinematically allowed. The decay modes are $\chi^{0} \rightarrow u d d \overline{\tilde{X}} \overline{\tilde{X}}$ or $\bar{u} \bar{d} \bar{d} \tilde{X} \tilde{X}$ followed by $\overline{\tilde{X}} \rightarrow X \bar{u} \bar{d} \bar{d}$ or $\tilde{X} \rightarrow \bar{X} u d d$. The decay length to $X$ scalars is

$$
\begin{aligned}
c \tau\left(\chi^{0} \rightarrow q q q \tilde{X} \tilde{X}\right) \sim & 0.3 \mathrm{~mm}\left(\frac{M}{\mathrm{TeV}}\right)^{4}\left(\frac{m}{500 \mathrm{GeV}}\right)^{4} \\
& \times\left(\frac{m_{\chi^{0}}}{100 \mathrm{GeV}}\right)^{-9} .
\end{aligned}
$$

This is followed by the $\tilde{X}$ decay with

$$
\begin{aligned}
c \tau(\tilde{X} \rightarrow X q q q) \sim & 3 \mathrm{~mm}\left(\frac{M}{\mathrm{TeV}}\right)^{4}\left(\frac{m}{500 \mathrm{GeV}}\right)^{2} \\
& \times\left(\frac{m_{\tilde{X}}}{100 \mathrm{GeV}}\right)^{-7} .
\end{aligned}
$$

We again emphasize that this involves crude estimates of the many-body phase space and loop factors. There are additional complications from the flavor structure of the interaction. If the dominant terms in Eq. (2.18) involve right-handed tops, there will be additional suppression if $\chi^{0}$ decays to tops are kinematically forbidden.

We now move onto the case of a squark LSP. If the $X$ scalars are light enough, the decay will go directly through the operator in Eq. (2.18) with decay length

$$
c \tau(\tilde{q} \rightarrow \tilde{X} \tilde{X} q q) \sim 10^{-8} \mathrm{~m}\left(\frac{M}{\mathrm{TeV}}\right)^{4}\left(\frac{m_{\tilde{q}}}{100 \mathrm{GeV}}\right)^{-5} .
$$

The $X$ scalars subsequently decay via Eq. (4.13). If the squark decay to $X$ scalars is not kinematically available, the decay must go through a squark loop with decay length

$$
\begin{aligned}
c \tau(\tilde{q} \rightarrow X X q q) \sim & 0.3 \mathrm{~cm}\left(\frac{M}{\mathrm{TeV}}\right)^{4}\left(\frac{m}{500 \mathrm{GeV}}\right)^{2} \\
& \times\left(\frac{m_{\tilde{q}}}{100 \mathrm{GeV}}\right)^{-7},
\end{aligned}
$$

where $m$ is an assumed common mass in the loop.

Finally we consider the case of a slepton LSP. The decay must go via a virtual gaugino $\tilde{\ell} \rightarrow \ell \chi^{*}$. The virtual gaugino has the same decay modes as the real gauginos discussed above. Thus through a neutralino, we may have $\tilde{\ell} \rightarrow$ eqqqXX with decay length

$$
\begin{aligned}
c \tau(\tilde{\ell} \rightarrow \ell q q q X X) \sim & 10^{5} \mathrm{~m}\left(\frac{M}{\mathrm{TeV}}\right)^{4}\left(\frac{m}{500 \mathrm{GeV}}\right)^{8} \\
& \times\left(\frac{m_{\tilde{\ell}}}{100 \mathrm{GeV}}\right)^{-13} .
\end{aligned}
$$

If the $X$ scalars are light enough, the slepton can decay via $\tilde{\ell} \rightarrow \ell q q q \tilde{X} \tilde{X}$ with decay length

$$
\begin{aligned}
c \tau(\tilde{\ell} \rightarrow \ell q q q \tilde{X} \tilde{X}) \sim & 1 \mathrm{~m}\left(\frac{M}{\mathrm{TeV}}\right)^{4}\left(\frac{m}{500 \mathrm{GeV}}\right)^{6} \\
& \times\left(\frac{m_{\tilde{\ell}}}{100 \mathrm{GeV}}\right)^{-11} .
\end{aligned}
$$

Again, these are highly uncertain estimates.

In several of the scenarios discussed above, the $X$ fermions and/or scalars can have lifetimes on cosmological time scales. This may be due either to larger values of $M$, different superpartner masses, or simply because of large suppression factors missed in the crude approximations made above. Such decays can have important effects on nucleosynthesis, matter-radiation equality, or the dark matter content of the Universe, among other issues. The study of these issues is beyond the scope of this work.

Finally, a few comments on the $L=1$ model. In this case the operator in Eq. (2.24) does not break $R$ parity so that both the LSP and the dark matter particle may be stable. In the non-SUSY UV completion of this model, there is some novel phenomenology associated with the $H^{\prime}$ state. As already mentioned in Sec. II C, the charged Higgs can be pair produced. They then decay via $H^{\prime \pm} \rightarrow \tau^{ \pm}+$ $\mathbb{E}_{T}$, the missing energy being carried away by dark matter.

\section{CONCLUSIONS}

We have presented a simple class of models in which the dark matter relic abundance is determined by the baryon asymmetry. This naturally explains the fact that the observed baryon and dark matter abundances are close, $\Omega_{\mathrm{DM}} \simeq 5 \Omega_{B}$. Conceptually these models are very simple: higher-dimension operators distribute the primordial $B-$ $L$ asymmetry between the dark and visible sectors. When these higher-dimension operators fall out of equilibrium, the asymmetry is separately frozen into the two sectors. We presented several simple examples as existence proofs. In any specific model, the dark matter mass is precisely 
determined, and we find masses in the range 5 to $15 \mathrm{GeV}$ in the models presented here.

The symmetric component of the dark matter abundance can be annihilated away either by some of the same interactions that transfer the asymmetry, or by the interactions that generate the dark matter mass. Interestingly, either of these mechanisms also gives a possible direct detection cross section large enough to be observed in upcoming experiments. This gives a strong motivation for additional dark matter experiments and analyses sensitive to dark matter in the low mass range. An interesting feature in the second class of models is that the dominant direct detection mechanism is through the electric charge radius of the dark matter.

This dark matter mechanism described here can be naturally combined with supersymmetry, giving a connection to a plausible model of electroweak physics and the hierarchy problem. In some models, the would-be LSP (and dark matter candidate) of supersymmetry naturally decays into pairs of dark matter particles. This gives rise to interesting modifications of standard supersymmetric collider phenomenology, including the possibility of highly displaced vertices.

This class of models gives a compelling alternative to the usual WIMP paradigm for dark matter that is worthy of further investigation.

\section{ACKNOWLEDGMENTS}

We thank G. Kribs, A. Kusenko, and J. Terning for discussions. We thank the Kavli Institute for Theoretical Physics and the Aspen Center for Physics, where part of this work was performed. This work was supported by the National Science Foundation under Grant No. NSF-PHY0401513 (D. E. K.) and by the U.S. Department of Energy, including Grant No. DE-FG02-95ER40896 and by NASA Grant No. NAG5-18042 (K. M.Z.).
[1] See e.g. G. Bertone, D. Hooper, and J. Silk, Phys. Rep. 405, 279 (2005).

[2] See e.g. C. Amsler et al. (Particle Data Group), Phys. Lett. B 667, 1 (2008).

[3] A. D. Sakharov, Pis'ma Zh. Eksp. Teor. Fiz. 5, 32 (1967) [JETP Lett. 5, 32 (1967)]; JETP Lett. 5, 24 (1967); Usp. Fiz. Nauk 161, 61 (1991); Sov. Phys. Usp. 34, 392 (1991).

[4] D. B. Kaplan, Phys. Rev. Lett. 68, 741 (1992).

[5] S. Nussinov, Phys. Lett. 165B, 55 (1985); S. M. Barr, Phys. Rev. D 44, 3062 (1991); S. M. Barr, R.S. Chivukula, and E. Farhi, Phys. Lett. B 241, 387 (1990); S. B. Gudnason, C. Kouvaris, and F. Sannino, Phys. Rev. D 73, 115003 (2006).

[6] S. Dodelson, B. R. Greene, and L. M. Widrow, Nucl. Phys. B372, 467 (1992).

[7] V. Kuzmin, Phys. Part. Nucl. 29, 257 (1998); Fiz. Elem. Chastits At. Yadra 29, 637 (1998) [Sov. J. Part. Nucl. 29, 637 (1998)]; Phys. At. Nucl. 61, 1107 (1998).

[8] M. Fujii and T. Yanagida, Phys. Lett. B 542, 80 (2002).

[9] R. Kitano and I. Low, Phys. Rev. D 71, 023510 (2005); R. Kitano, H. Murayama, and M. Ratz, Phys. Lett. B 669, 145 (2008).

[10] G. R. Farrar and G. Zaharijas, Phys. Rev. Lett. 96, 041302 (2006).

[11] D. Hooper, J. March-Russell, and S. M. West, Phys. Lett. B 605, 228 (2005)

[12] L. Roszkowski and O. Seto, Phys. Rev. Lett. 98, 161304 (2007); O. Seto and M. Yamaguchi, Phys. Rev. D 75, 123506 (2007).

[13] R. Bernabei et al. (DAMA Collaboration), Eur. Phys. J. C 56, 333 (2008).

[14] P. Gondolo and G. Gelmini, Phys. Rev. D 71, 123520 (2005).

[15] A. Bottino, N. Fornengo, and S. Scopel, Phys. Rev. D 67,
063519 (2003); A. Bottino, F. Donato, N. Fornengo, and S. Scopel, Phys. Rev. D 77, 015002 (2008).

[16] F. Petriello and K. M. Zurek, J. High Energy Phys. 09 (2008) 047.

[17] C. Savage, G. Gelmini, P. Gondolo, and K. Freese, J. Cosmol. Astropart. Phys. 04 (2009) 010; C. Savage, K. Freese, P. Gondolo, and D. Spolyar, arXiv:0901.2713.

[18] S. Chang, A. Pierce, and N. Weiner, arXiv:0808.0196; M. Fairbairn and T. Schwetz, J. Cosmol. Astropart. Phys. 01 (2009) 037.

[19] D. Hooper, F. Petriello, K. M. Zurek, and M. Kamionkowski, Phys. Rev. D 79, 015010 (2009).

[20] J. A. Harvey and M. S. Turner, Phys. Rev. D 42, 3344 (1990).

[21] P. Arnold and L. D. McLerran, Phys. Rev. D 36, 581 (1987).

[22] R. Harnik, G. D. Kribs, D. T. Larson, and H. Murayama, Phys. Rev. D 70, 015002 (2004); S. Chang, C. Kilic, and R. Mahbubani, Phys. Rev. D 71, 015003 (2005); A. Delgado and T.M.P. Tait, J. High Energy Phys. 07 (2005) 023.

[23] R. Dermisek and J. F. Gunion, Phys. Rev. Lett. 95, 041801 (2005); S. Chang, P. J. Fox, and N. Weiner, J. High Energy Phys. 08 (2006) 068; P. W. Graham, A. Pierce, and J. G. Wacker, arXiv:hep-ph/0605162.

[24] G. F. Giudice and A. Masiero, Phys. Lett. B 206, 480 (1988).

[25] P. Garcia-Abia (LEP Collaborations), The 32nd International Conference on High-Energy Physics (ICHEP 04), Beijing, China, 2004 (World Scientific, Singapore, 2005).

[26] M. J. Strassler and K. M. Zurek, Phys. Lett. B 651, 374 (2007); M. J. Strassler and K. M. Zurek, Phys. Lett. B 661, 263 (2008). 
[27] J. Kang and M. A. Luty, arXiv:0805.4642.

[28] J. Angle et al. (XENON Collaboration), Phys. Rev. Lett. 100, 021303 (2008).

[29] Z. Ahmed et al. (CDMS Collaboration), Phys. Rev. Lett. 102, 011301 (2009).

[30] S. Gardner, Phys. Rev. D 79, 055007 (2009).
[31] S. Raby and G. West, Phys. Lett. B 200, 547 (1988).

[32] S. Chang, R. Dermisek, J.F. Gunion, and N. Weiner, Annu. Rev. Nucl. Part. Sci. 58, 75 (2008).

[33] H. C. Cheng, J. F. Gunion, Z. Han, G. Marandella, and B. McElrath, J. High Energy Phys. 12 (2007) 076. 